\title{
n-Tupled Fixed Points Theorem in Fuzzy Metric Spaces with Application
}

\author{
P. P. Murthy and Rashmi Kenvat \\ Department of Pure and Applied Mathematics, Guru Ghasidas Vishwavidyalaya (A Central University), Koni, \\ Bilaspur, Chhattisgarh 495 009, India
}

Correspondence should be addressed to Rashmi Kenvat; rashmikwt@gmail.com

Received 17 July 2014; Revised 16 January 2015; Accepted 8 February 2015

Academic Editor: Ning Xiong

Copyright (C) 2015 P. P. Murthy and R. Kenvat. This is an open access article distributed under the Creative Commons Attribution License, which permits unrestricted use, distribution, and reproduction in any medium, provided the original work is properly cited.

We will introduce the concept of $n$-tupled fixed points (for positive integer $n$ ) in fuzzy metric space by mild modification of the concept of $n$-tupled fixed points (for even positive interger $n$ ) introduced by Imdad et al. (2013) in metric spaces. As application of the above-mentioned concept, we will establish some $n$-tupled fixed point theorems for contractive type mappings in fuzzy metric space which extends the result of Roldán et al. (2013). Also we have given an application to solve a kind of Lipschitzian systems for $n$ variables and an integral system.

\section{Introduction}

The concept of coupled fixed point was introduced by Bhaskar and Lakshmikantham [1] and it motivated the fixed point theorists to work in the area of multidimensional fixed points; for example, see [2-9].

In recent years some of the fixed point theorists tried to establish the existence of $n$-tupled fixed points and common $n$-tupled fixed points for some contractions in metric spaces, partially ordered metric spaces and asymptotically regular metric spaces. In particular, Imdad et al. [9] introduced the concept of $n$-tupled coincidence points as well as $n$-tupled fixed point (for even positive integer) and utilize these two definitions to obtain $n$-tupled coincidence as well as $n$-tupled common fixed point theorems. Very recently, Soliman et al. [10] proved some $n$-tupled coincident point theorems in partially ordered complete asymptotically regular metric spaces.

The purpose of our results is to introduce $n$-tupled fixed points (for all positive integers) and to prove $n$-tupled fixed points theorems for contractive type mappings in fuzzy metric spaces. Results obtained by us in Section 2 extents the work of Roldán et al. [11]. At the end we have given an application to solve a kind of Lipschitzian systems for $n$ variables and an integral system. Results proved in this paper follow the lines of the proof of Roldán et al. [11] for obtaining coincidence and fixed points.

A triangular norm (also called a $t$-norm) is a mapping * : $[0,1] \times[0,1] \rightarrow[0,1]$ is associative, commutative, and nondecreasing in both arguments and has 1 as an identity element.

Definition 1 (see $[11,12])$. For any $a \in[0,1]$, let the sequence $\left\{*^{n} a\right\}_{n=1}^{\infty}$ be defined by $*^{1} a=a$ and $*^{n} a=\left(*^{n-1} a\right) * a$. Then a $t$-norm $*$ is said to be of $H$-type if the sequence $\left\{*^{n} a\right\}_{n=1}^{\infty}$ is equicontinuous at $a=1$, that is, for all $\epsilon \in(0,1)$, there exists $\eta \in(0,1)$ such that if $a \in(1-\eta, 1]$, then $*^{m} a>1-\epsilon$ for all $m \in N$.

The most important and well-known continuous $t$-norm of $H$-type is $*=\min$, which verifies $\min (a, b) \geq a b$ for all $a, b \in[0,1]$. The following result presents a wide range of $t$ norms of $H$-type.

Lemma 2. Let $\delta \in(0,1]$ be a real number and let $*$ be a $t$ norm. Define $*_{\delta}$ as $x *_{\delta} y=x * y$, if $\max (x, y) \leq 1-\delta$ and $x *_{\delta} y=\min (x, y)$, if $\max (x, y)>1-\delta$. Then $*_{\delta}$ is a t-norm of H-type. 
Definition 3 (see [13]). A triplet $(X, M, *)$ is said to be a fuzzy metric space (in the sense of Kramosil and Michalek; briefly, a FMS), if $X$ is a nonempty set, $*$ is a continuous $t$-norm, and $M: X \times X \times[0, \infty) \rightarrow[0,1]$ is a fuzzy set satisfying the following conditions for each $x, y, z \in X$ and $t, s>0$ :

$\left(\mathrm{FM}_{1}\right) M(x, y, t)>0$,

$\left(\mathrm{FM}_{2}\right) M(x, y, t)=1$ if and only if $x=y$,

$\left(\mathrm{FM}_{3}\right) M(x, y, t)=M(y, x, t)$,

$\left(\mathrm{FM}_{4}\right) M(x, y, \cdot):[0, \infty) \rightarrow[0,1]$ is left continuous,

$\left(\mathrm{FM}_{5}\right) M(x, y, t) * M(y, z, s) \leq M(x, z, t+s)$,

in this case, we also say that $(X, M)$ is a FMS under $*$; in the sequel, we will only consider FMS verifying

$\left(\mathrm{FM}_{6}\right) \lim _{t \rightarrow \infty} M(x, y, t)=1$ for all $x, y \in X$.

Lemma 4. $M(x, y, \cdot)$ is a nondecreasing function on $[0, \infty)$.

Definition 5 (see [14]). Let $(X, M, *)$ be a fuzzy metric space; then

(1) a sequence $\left\{x_{n}\right\}$ in $X$ is said to be convergent to $x$ if

$$
\lim _{n \rightarrow \infty} M\left(x_{n}, x, t\right)=1
$$

for all $t>0$;

(2) a sequence $\left\{x_{n}\right\}$ in $X$ is said to be a Cauchy sequence if, for any $\epsilon>0$, there exists $n_{0} \in \mathbb{N}$, such that

$$
M\left(x_{n}, x_{m}, t\right)>1-\epsilon
$$

for all $t>0$ and $n, m \geq n_{0}$;

(3) a fuzzy metric space $(X, M, *)$ is said to be complete if and only if every Cauchy sequence in $X$ is convergent.

Given any $t$-norm $*$, it is easy to prove that $* \leq \min$. Therefore, if $(X, M)$ is a FMS under min, then $(X, M)$ is a FMS under any (continuous or not) $t$-norm. This is the case in the following examples (in which, obviously, we only define $M(x, y, t)$ for $t>0$ and $x \neq y)$.

Example 6. From a metric space $(X, d)$, we can consider a FMS in different ways. For $t>0$ and $x \neq y$, define

(i) $M_{d}(x, y, t)=t /(t+d(x, y))$;

(ii) $M_{e}(x, y, t)=e^{-(d(x, y) / t)}$;

(iii) $M_{c}(x, y, t)=\left\{\begin{array}{c}0, \text { if } t \leq d(x, y) \\ 1 \text { if } t>d(x, y) .\end{array}\right.$

It is well known that $\left(X, M_{d}\right)$ is a FMS under the product $*=$ $\cdot$, called the standard FMS on $(X, d)$, since it is the standard way of viewing the metric space $(X, d)$ as a FMS. However, it is also true (though lesser known) that $\left(X, M_{d}\right),\left(X, M_{e}\right)$, and $\left(X, M_{c}\right)$ are FMS under min.

Furthermore, $(X, d)$ is a complete metric space if and only if $\left(X, M_{d}\right)$ (or $\left(X, M_{c}\right)$ or $\left.\left(X, M_{e}\right)\right)$ is a complete FMS. For instance, this is the case of any nonempty and closed subset (or subinterval) of $R$ provided with its Euclidean metric.
Definition 7. A function $g: X \rightarrow X$ on a fuzzy metric space is said to be continuous at a point $x_{0} \in X$ if, for any sequence $\left\{x_{n}\right\}$ in $X$ converging to $x_{0}$, the sequence $\left\{g x_{n}\right\}$ converges to $g x_{0}$. If $g$ is continuous at each $x \in X$, then $g$ is said to be continuous on $X$. As usual, if $x_{0} \in X$, we will denote $g^{-1}\left(x_{0}\right)=\left\{x \in X: g x=x_{0}\right\}$.

Remark 8. If $x \in[0,1]$ and $a, b \in(0, \infty)$, then $a \leq b$ implies that $x^{a} \geq x^{b}$. We will use this fact in the following way: $0<a \leq b \leq 1$ implies that $[M(x, y, t)]^{a} \geq[M(x, y, t)]^{b} \geq$ $M(x, y, t)$.

\section{Main Results}

Henceforth, $X$ will denote a nonempty set and $X^{n}=$ $\underbrace{X \times X \times \cdots \times X}$.

$n$-times

Here, we introduce $n$-tupled fixed points (for positive integer $n$ ) in fuzzy metric space by slightly modified concept of $n$-tupled fixed points (for natural number $n$ ) introduced by Imdad et al. [9] in metric space.

Definition 9. Let $F: X^{n} \rightarrow X$ and $g: X \rightarrow X$ be two mappings.

(a) One says that $F$ and $g$ are commuting if

$$
g F\left(x^{(1)}, x^{(2)}, \ldots, x^{(n)}\right)=F\left(g x^{(1)}, g x^{(2)}, \ldots, g x^{(n)}\right)
$$

for all $x^{(1)}, x^{(2)}, \ldots, x^{(n)} \in X$.

(b) An element $\left(x^{(1)}, x^{(2)}, x^{(3)}, \ldots, x^{(n)}\right) \in X^{n}$ is called $n$ tupled coincidence points of the mappings $F$ and $g$ if

$$
\begin{gathered}
F\left(x^{(1)}, x^{(2)}, x^{(3)}, \ldots, x^{(n)}\right)=g x^{(1)} \\
F\left(x^{(2)}, x^{(3)}, \ldots, x^{(n)}, x^{(1)}\right)=g x^{(2)} \\
F\left(x^{(3)}, \ldots, x^{(n)}, x^{(1)}, x^{(2)}\right)=g x^{(3)} \\
\vdots \\
F\left(x^{(n)}, x^{(1)}, x^{(2)}, \ldots, x^{(n-1)}\right)=g x^{(n)} .
\end{gathered}
$$

Definition 10. An element $\left(x^{(1)}, x^{(2)}, \ldots, x^{(n)}\right) \in X^{n}$ is called an $n$-tupled fixed point of map $F: X^{n} \rightarrow X$ and $g: X \rightarrow X$ if

$$
\begin{gathered}
F\left(x^{(1)}, x^{(2)}, x^{(3)}, \ldots, x^{(n)}\right)=g x^{(1)}=x^{(1)} \\
F\left(x^{(2)}, x^{(3)}, \ldots, x^{(n)}, x^{(1)}\right)=g x^{(2)}=x^{(2)} \\
F\left(x^{(3)}, \ldots, x^{(n)}, x^{(1)}, x^{(2)}\right)=g x^{(3)}=x^{(3)} \\
\vdots \\
F\left(x^{(n)}, x^{(1)}, x^{(2)}, \ldots, x^{(n-1)}\right)=g x^{(n)}=x^{(n)} .
\end{gathered}
$$

Now, we present our main theorem. 
Theorem 11. Let $*$ be a $t$-norm of $H$-type such that $s * t \geq$ st for all $s, t \in[0,1]$. Let $k \in(0,1)$ and $a_{1}, a_{2}, \ldots, a_{n} \in$ $[0,1]$ be real numbers such that $a_{1}+a_{2}+\cdots+a_{n} \leq 1$, let $(X, M, *)$ be a complete FMS, and let $F: X^{n} \rightarrow X$ and $g: X \rightarrow X$ be two mappings such that $F\left(X^{n}\right) \subseteq g(X)$ and $g$ is continuous and commuting with $F$. Suppose that, for all $x^{(1)}, x^{(2)}, \ldots, x^{(n)}, y^{(1)}, y^{(2)}, \ldots, y^{(n)} \in X$ and all $t>0$,

$$
\begin{aligned}
M( & F\left(x^{(1)}, x^{(2)}, \ldots, x^{(n)}\right), \\
& \left.F\left(y^{(1)}, y^{(2)}, \ldots, y^{(n)}\right), k t\right) \\
\geq & {\left[M\left(g x^{(1)}, g y^{(1)}, t\right)\right]^{a_{1}} } \\
& *\left[M\left(g x^{(2)}, g y^{(2)}, t\right)\right]^{a_{2}} * \ldots \\
& *\left[M\left(g x^{(n)}, g y^{(n)}, t\right)\right]^{a_{n}} .
\end{aligned}
$$

Then there exists a unique $x^{(1)} \in X$ such that $x^{(1)}=g x^{(1)}=$ $F\left(x^{(1)}, x^{(1)}, \ldots, x^{(1)}\right)$. In particular, $F$ and $g$ have, at least, one $n$-tupled coincidence point. Furthermore, $\left(x^{(1)}, x^{(1)}, \ldots, x^{(1)}\right)$ is the unique $n$-tupled coincidence point of $F$ and $g$ if we assume that $g^{-1}\left(x_{0}^{(1)}\right)=\left\{x_{0}^{(1)}\right\}$ only in the case that $F \equiv x_{0}^{(1)}$ is constant on $X^{n}$.

In this result, in order to avoid the indetermination $0^{0}$, we assume that $\left[M\left(g x^{(1)}, g x^{(2)}, t\right)\right]^{0}=1$ for all $t>0$ and all $x^{(1)}, x^{(2)} \in X$.

Proof. Suppose that $F$ is constant in $X^{n}$; that is, there exists $x_{0}^{(1)} \in X$ such that

$$
F\left(x^{(1)}, x^{(2)}, \ldots, x^{(n)}\right)=x_{0}^{(1)} \quad \forall x^{(1)}, x^{(2)}, \ldots, x^{(n)} \in X .
$$

As $F$ and $g$ are commuting, we deduce that

$$
\begin{aligned}
g x_{0}^{(1)} & =g F\left(x^{(1)}, x^{(2)}, \ldots, x^{(n)}\right) \\
& =F\left(g x^{(1)} g x^{(2)} \cdots g x^{(n)}\right)=x_{0}^{(1)} .
\end{aligned}
$$

Therefore, $x_{0}^{(1)}=g x_{0}^{(1)}=F\left(x_{0}^{(1)}, x_{0}^{(1)}, \ldots, x_{0}^{(1)}\right)$ and $\left(x_{0}^{(1)}, x_{0}^{(1)}\right.$, $\left.\ldots, x_{0}^{(1)}\right)$ is $n$-tupled coincidence point of $F$ and $g$.

Now, suppose that $g^{-1}\left(x_{0}^{(1)}\right)=\left\{x_{0}^{(1)}\right\}$ and $\left(x^{(1)}, x^{(2)}\right.$, $\left.\ldots, x^{(n)}\right) \in X^{n}$ is another $n$-tupled coincidence point of $F$ and g. Then,

$$
\begin{aligned}
g x^{(1)}= & F\left(x^{(1)}, x^{(2)}, \ldots, x^{(n)}\right)=x_{0}^{(1)}, \\
& \text { so } x^{(1)} \in g^{-1}\left(x_{0}^{(1)}\right)=\left\{x_{0}^{(1)}\right\} .
\end{aligned}
$$

Similarly, $x^{(1)}=x^{(2)}=\cdots=x^{(n)}=x_{0}^{(1)}$ and $\left(x_{0}^{(1)}, x_{0}^{(1)}, \ldots\right.$, $\left.x_{0}^{(1)}\right)$ is the unique $n$-tupled coincidence points of $F$ and $g$.

Next, suppose that $F$ is not constant in $X^{n}$.

In this case, $\left(x^{(1)}, x^{(2)}, \ldots, x^{(n)}\right) \neq(0,0,0)$ and the proof is divided into five steps. In the entire proof, $n$ and $p$ stand to be nonnegative integers and $t \in[0, \infty)$.

Step 1. First we define of the sequences $\left\{x_{n}^{(1)}\right\},\left\{x_{n}^{(2)}\right\}, \ldots,\left\{x_{n}^{(n)}\right\}$.
Let $x_{0}^{(1)}, x_{0}^{(2)}, \ldots, x_{0}^{(n)} \in X$ be arbitrary points in $X$.

Since $F\left(X^{n}\right) \subseteq g(X)$. we can choose $x_{1}^{(1)}, x_{1}^{(2)}, \ldots, x_{1}^{(n)} \in$ $X$ such that

$$
\begin{gathered}
g\left(x_{1}^{(1)}\right)=F\left(x_{0}^{(1)}, x_{0}^{(2)}, \ldots, x_{0}^{(n)}\right), \\
g\left(x_{1}^{(2)}\right)=F\left(x_{0}^{(2)}, x_{0}^{(3)}, \ldots, x_{0}^{(n)}, x_{0}^{(1)}\right), \\
\vdots \\
g\left(x_{1}^{(n)}\right)=F\left(x_{0}^{(n)}, x_{0}^{(1)}, \ldots, x_{0}^{(n-1)}\right),
\end{gathered}
$$

Again from $F\left(X^{n}\right) \subseteq g(X)$. we can choose $x_{2}^{(1)}, x_{2}^{(2)}, \ldots, x_{2}^{(n)} \in$ $X$ such that

$$
\begin{gathered}
g\left(x_{2}^{(1)}\right)=F\left(x_{1}^{(1)}, x_{1}^{(2)}, \ldots, x_{1}^{(n)}\right), \\
g\left(x_{2}^{(2)}\right)=F\left(x_{1}^{(2)}, x_{1}^{(3)}, \ldots, x_{1}^{(n)}, x_{1}^{(1)}\right), \\
\vdots \\
g\left(x_{2}^{(n)}\right)=F\left(x_{1}^{(n)}, x_{1}^{(1)}, \ldots, x_{1}^{(n-1)}\right),
\end{gathered}
$$

Continuing in this way, we can construct sequences $\left\{x_{n}^{(1)}\right\},\left\{x_{n}^{(2)}\right\}, \ldots,\left\{x_{n}^{(n)}\right\}$ in $X$ such that

$$
\begin{gathered}
g\left(x_{n}^{(1)}\right)=F\left(x_{n-1}^{(1)}, x_{n-1}^{(2)}, \ldots, x_{n-1}^{(n)}\right), \\
g\left(x_{n}^{(2)}\right)=F\left(x_{n-1}^{(2)}, x_{n-1}^{(3)}, \ldots, x_{n-1}^{(n)}, x_{n-1}^{(1)}\right), \\
\vdots \\
g\left(x_{n}^{(n)}\right)=F\left(x_{n-1}^{(n)}, x_{n-1}^{(1)}, \ldots, x_{n-1}^{(n-1)}\right),
\end{gathered}
$$

for $n \geq 1$.

Step 2. Now, we will show that the sequences $\left\{g x_{n}^{(1)}\right\},\left\{g x_{n}^{(2)}\right\}$, $\ldots,\left\{g x_{n}^{(n)}\right\}$ are Cauchy sequences. Define, for $n \geq 1$ and all $t \geq 0$,

$$
\begin{aligned}
\delta_{n}(t)= & M\left(g\left(x_{n}^{(1)}\right), g\left(x_{n+1}^{(1)}\right), t\right) \\
& * M\left(g\left(x_{n}^{(2)}\right), g\left(x_{n+1}^{(2)}\right), t\right) \\
& * \cdots * M\left(g\left(x_{n}^{(n)}\right), g\left(x_{n+1}^{(n)}\right), t\right) .
\end{aligned}
$$

Since $\delta_{n}$ is a nondecreasing function and $t-k t \leq t \leq t / k$, we have that

$$
\delta_{n}(t-k t) \leq \delta_{n}(t) \leq \delta_{n}\left(\frac{t}{k}\right), \quad \forall t>0, n \geq 1 .
$$

From inequality (6) we deduce, for all $n \in N$ and all $t \geq 0$,

$$
\begin{aligned}
& M\left(g\left(x_{n}^{(1)}\right), g\left(x_{n+1}^{(1)}\right), t\right) \\
& =M\left(F\left(x_{n-1}^{(1)}, x_{n-1}^{(2)}, \ldots, x_{n-1}^{(n)}\right),\right. \\
& \left.\quad F\left(x_{n}^{(1)}, x_{n}^{(2)}, \ldots, x_{n}^{(n)}\right), t\right)
\end{aligned}
$$




$$
\begin{aligned}
\geq & {\left[M\left(g x_{n-1}^{(1)}, g x_{n}^{(1)}, \frac{t}{k}\right)\right]^{a_{1}} } \\
& *\left[M\left(g x_{n-1}^{(2)} g x_{n}^{(2)}, \frac{t}{k}\right)\right]^{a_{2}} \\
& * \cdots *\left[M\left(g x_{n-1}^{(n)}, g x_{n}^{(n)}, \frac{t}{k}\right)\right]^{a_{n}} \\
M( & \left.g\left(x_{n}^{(2)}\right), g\left(x_{n+1}^{(2)}\right), t\right) \\
= & M\left(F\left(x_{n-1}^{(2)}, x_{n-1}^{(3)}, \ldots, x_{n-1}^{(1)}\right),\right. \\
\geq & {\left[F\left(x_{n}^{(2)}, x_{n}^{(3)}, \ldots, x_{n}^{(1)}\right), t\right) } \\
\geq & *\left[M\left(g x_{n-1}^{(2)}, g x_{n}^{(2)}, \frac{t}{k}\right)\right]^{a_{1}} \\
& \left.* \cdots\left(g x_{n-1}^{(n)}, g x_{n}^{(3)}, \frac{t}{k}\right)\right]^{a_{2}} \\
& * \cdots\left(M\left(g x_{n-1}^{(1)} g x_{n}^{(1)}, \frac{t}{k}\right)\right]^{a_{2}} \\
& \left.\left.\left.* M\left(g x_{n}^{(n-1)}, g x_{n}^{(1)}, \frac{t}{k}\right)\right]^{a_{n}}, g x_{n}^{(1)}, \frac{t}{k}\right)\right]^{a_{n}} \\
M( & \left.M\left(x_{n}^{(n)}\right), g\left(x_{n+1}^{(n)}\right), t\right) \\
& \left.M\left(x_{n-1}^{(n)}, x_{n-1}^{(1)}, \ldots, x_{n-1}^{(1)}, \ldots, x_{n}^{(n-1)}\right), t\right) \\
& \quad \cdots
\end{aligned}
$$

Using (15) and Remark 8, we have

$$
\begin{aligned}
M( & \left.g\left(x_{n}^{(1)}\right), g\left(x_{n+1}^{(1)}\right), t\right) \\
\geq & {\left[M\left(g x_{n-1}^{(1)}, g x_{n}^{(1)}, \frac{t}{k}\right)\right]^{a_{1}} } \\
& *\left[M\left(g x_{n-1}^{(2)} g x_{n}^{(2)}, \frac{t}{k}\right)\right]^{a_{2}} \\
& * \cdots *\left[M\left(g x_{n-1}^{(n)}, g x_{n}^{(n)}, \frac{t}{k}\right)\right]^{a_{n}} \\
\geq & M\left(g x_{n-1}^{(1)}, g x_{n}^{(1)}, \frac{t}{k}\right) * M\left(g x_{n-1}^{(2)}, g x_{n}^{(2)}, \frac{t}{k}\right) \\
& * \cdots * M\left(g x_{n-1}^{(n)}, g x_{n}^{(n)}, \frac{t}{k}\right) \\
= & \delta_{n-1}\left(\frac{t}{k}\right)
\end{aligned}
$$

$$
\begin{aligned}
& M\left(g\left(x_{n}^{(2)}\right), g\left(x_{n+1}^{(2)}\right), t\right) \\
& \geq\left[M\left(g x_{n-1}^{(2)}, g x_{n}^{(2)}, \frac{t}{k}\right)\right]^{a_{1}} \\
& *\left[M\left(g x_{n-1}^{(3)}, g x_{n}^{(3)}, \frac{t}{k}\right)\right]^{a_{2}} \\
& * \cdots *\left[M\left(g x_{n-1}^{(1)}, g x_{n}^{(1)}, \frac{t}{k}\right)\right]^{a_{n}} \\
& \geq M\left(g x_{n-1}^{(2)}, g x_{n}^{(2)}, \frac{t}{k}\right) \\
& * M\left(g x_{n-1}^{(3)}, g x_{n}^{(3)}, \frac{t}{k}\right) \\
& * \cdots * M\left(g x_{n-1}^{(1)}, g x_{n}^{(1)}, \frac{t}{k}\right) \\
& =\delta_{n-1}\left(\frac{t}{k}\right) \\
& M\left(g\left(x_{n}^{(n)}\right), g\left(x_{n+1}^{(n)}\right), t\right) \\
& \geq\left[M\left(g x_{n-1}^{(n)}, g x_{n}^{(n)}, \frac{t}{k}\right)\right]^{a_{1}} \\
& \text { * }\left[M\left(g x_{n-1}^{(1)}, g x_{n}^{(1)}, \frac{t}{k}\right)\right]^{a_{2}} \\
& * \cdots *\left[M\left(g x_{n-1}^{(n-1)}, g x_{n}^{(n-1)}, \frac{t}{k}\right)\right]^{a_{n}} \\
& \geq M\left(g x_{n-1}^{(n)}, g x_{n}^{(n)}, \frac{t}{k}\right) \\
& * M\left(g x_{n-1}^{(1)}, g x_{n}^{(1)}, \frac{t}{k}\right) \\
& * \cdots * M\left(g x_{n-1}^{(n-1)}, g x_{n}^{(n-1)}, \frac{t}{k}\right) \\
& =\delta_{n-1}\left(\frac{t}{k}\right) \text {. }
\end{aligned}
$$

This proves that, for all $t>0$ and all $n \geq 0$,

$$
\begin{aligned}
M( & \left.g\left(x_{n}^{(1)}\right), g\left(x_{n+1}^{(1)}\right), t\right) \\
& * M\left(g\left(x_{n}^{(2)}\right), g\left(x_{n+1}^{(2)}\right), t\right) \\
& * \cdots * M\left(g\left(x_{n}^{(n)}\right), g\left(x_{n+1}^{(n)}\right), t\right) \\
\geq & \delta_{n-1}\left(\frac{t}{k}\right) \geq \delta_{n-1}(t) .
\end{aligned}
$$

Swapping $t$ by $t-k t$, we deduce, for all $t>0$ and $n \geq 0$, that

$$
\begin{aligned}
& M\left(g\left(x_{n}^{(1)}\right), g\left(x_{n+1}^{(1)}\right),(t-k t)\right) \\
& \quad * M\left(g\left(x_{n}^{(2)}\right), g\left(x_{n+1}^{(2)}\right),(t-k t)\right) \\
& \quad * \cdots * M\left(g\left(x_{n}^{(n)}\right), g\left(x_{n+1}^{(n)}\right),(t-k t)\right) \\
& \geq \delta_{n-1}(t-k t) .
\end{aligned}
$$


Taking into account that $*$ is commutative and $* \geq \cdot$ and (15), we observe that

$$
\begin{aligned}
& \delta_{n}(t)=M\left(g\left(x_{n}^{(1)}\right), g\left(x_{n+1}^{(1)}\right), t\right) \\
& \text { * } M\left(g\left(x_{n}^{(2)}\right), g\left(x_{n+1}^{(2)}\right), t\right) \\
& * \cdots * M\left(g\left(x_{n}^{(n)}\right), g\left(x_{n+1}^{(n)}\right), t\right) \\
& \geq\left[M\left(g x_{n-1}^{(1)}, g x_{n}^{(1)}, \frac{t}{k}\right)\right]^{a_{1}} \\
& \text { * }\left[M\left(g x_{n-1}^{(2)}, g x_{n}^{(2)}, \frac{t}{k}\right)\right]^{a_{2}} \\
& * \cdots *\left[M\left(g x_{n-1}^{(n)}, g x_{n}^{(n)}, \frac{t}{k}\right)\right]^{a_{n}} \\
& \text { * }\left[M\left(g x_{n-1}^{(2)}, g x_{n}^{(2)}, \frac{t}{k}\right)\right]^{a_{1}} \\
& \text { * }\left[M\left(g x_{n-1}^{(3)}, g x_{n}^{(3)}, \frac{t}{k}\right)\right]^{a_{2}} \\
& * \cdots *\left[M\left(g x_{n-1}^{(1)}, g x_{n}^{(1)}, \frac{t}{k}\right)\right]^{a_{n}} \\
& * \cdots *\left[M\left(g x_{n-1}^{(n)}, g x_{n}^{(n)}, \frac{t}{k}\right)\right]^{a_{1}} \\
& \text { * }\left[M\left(g x_{n-1}^{(1)}, g x_{n}^{(1)}, \frac{t}{k}\right)\right]^{a_{2}} \\
& * \cdots *\left[M\left(g x_{n-1}^{(n-1)}, g x_{n}^{(n-1)}, \frac{t}{k}\right)\right]^{a_{n}} \\
& =\left[M\left(g x_{n-1}^{(1)}, g x_{n}^{(1)}, \frac{t}{k}\right)\right]^{a_{1}} \\
& \text { * }\left[M\left(g x_{n-1}^{(1)}, g x_{n}^{(1)}, \frac{t}{k}\right)\right]^{a_{2}} \\
& * \cdots *\left[M\left(g x_{n-1}^{(1)}, g x_{n}^{(1)}, \frac{t}{k}\right)\right]^{a_{n}} \\
& \text { * }\left[M\left(g x_{n-1}^{(2)}, g x_{n}^{(2)}, \frac{t}{k}\right)\right]^{a_{1}} \\
& \text { * }\left[M\left(g x_{n-1}^{(2)}, g x_{n}^{(2)}, \frac{t}{k}\right)\right]^{a_{2}} \\
& * \cdots *\left[M\left(g x_{n-1}^{(2)}, g x_{n}^{(2)}, \frac{t}{k}\right)\right]^{a_{n}} \\
& * \cdots *\left[M\left(g x_{n-1}^{(n)}, g x_{n}^{(n)}, \frac{t}{k}\right)\right]^{a_{1}} \\
& \text { * }\left[M\left(g x_{n-1}^{(n)}, g x_{n}^{(n)}, \frac{t}{k}\right)\right]^{a_{2}} \\
& * \cdots *\left[M\left(g x_{n-1}^{(n)}, g x_{n}^{(n)}, \frac{t}{k}\right)\right]^{a_{n}} \\
& \geq\left[M\left(g x_{n-1}^{(1)}, g x_{n}^{(1)}, \frac{t}{k}\right)\right]^{a_{1}} \\
& \cdot\left[M\left(g x_{n-1}^{(1)}, g x_{n}^{(1)}, \frac{t}{k}\right)\right]^{a_{2}} \\
& \cdot \ldots \cdot\left[M\left(g x_{n-1}^{(1)}, g x_{n}^{(1)}, \frac{t}{k}\right)\right]^{a_{n}} \\
& \text { * }\left[M\left(g x_{n-1}^{(2)}, g x_{n}^{(2)}, \frac{t}{k}\right)\right]^{a_{1}} \\
& \cdot\left[M\left(g x_{n-1}^{(2)}, g x_{n}^{(2)}, \frac{t}{k}\right)\right]^{a_{2}}
\end{aligned}
$$

$$
\begin{aligned}
& \ldots \cdot\left[M\left(g x_{n-1}^{(2)}, g x_{n}^{(2)}, \frac{t}{k}\right)\right]^{a_{n}} \\
& * \cdots *\left[M\left(g x_{n-1}^{(n)}, g x_{n}^{(n)}, \frac{t}{k}\right)\right]^{a_{1}} \\
& {\left[M\left(g x_{n-1}^{(n)}, g x_{n}^{(n)}, \frac{t}{k}\right)\right]^{a_{2}} } \\
& \ldots \cdot\left[M\left(g x_{n-1}^{(n)}, g x_{n}^{(n)}, \frac{t}{k}\right)\right]^{a_{n}} \\
= & {\left[M\left(g x_{n-1}^{(1)}, g x_{n}^{(1)}, \frac{t}{k}\right)\right]^{a_{1}+a_{2}+\cdots+a_{n}} } \\
& *\left[M\left(g x_{n-1}^{(2)}, g x_{n}^{(2)}, \frac{t}{k}\right)\right]^{a_{1}+a_{2}+\cdots+a_{n}} \\
& * \cdots *\left[M\left(g x_{n-1}^{(n)}, g x_{n}^{(n)}, \frac{t}{k}\right)\right]^{a_{1}+a_{2}+\cdots+a_{n}} \\
\geq & M\left(g x_{n-1}^{(1)}, g x_{n}^{(1)}, \frac{t}{k}\right) \\
& * M\left(g x_{n-1}^{(2)}, g x_{n}^{(2)}, \frac{t}{k}\right) \\
& * \cdots * M\left(g x_{n-1}^{(n)}, g x_{n}^{(n)}, \frac{t}{k}\right) \\
= & \delta_{n-1}\left(\frac{t}{k}\right) .
\end{aligned}
$$

If we join this property to (14),

$$
\begin{array}{r}
\delta_{n}(t) \geq \delta_{n-1}\left(\frac{t}{k}\right) \geq \delta_{n-1}(t) \geq \delta_{n-1}(t-k t), \\
\forall t>0, n \geq 1 .
\end{array}
$$

Repeatedly applying the first inequality, we deduce that

$$
\delta_{n}(t) \geq \delta_{n-1}\left(\frac{t}{k}\right) \geq \delta_{n-2}\left(\frac{t}{k^{2}}\right) \geq \cdots \geq \delta_{0}\left(\frac{t}{k^{n}}\right)
$$

for all $t>0$ and $n \geq 1$. This means that, for all $t>0$,

$$
\begin{aligned}
\lim _{n \rightarrow \infty} \delta_{n}(t) & \geq \lim _{n \rightarrow \infty} \delta_{0}\left(\frac{t}{k^{n}}\right)=1 \\
& \Longrightarrow \lim _{n \rightarrow \infty} \delta_{n}(t)=1 .
\end{aligned}
$$

Properties (17) and (20) imply that

$$
\begin{aligned}
M( & \left.g\left(x_{n}^{(1)}\right), g\left(x_{n+1}^{(1)}\right), t\right) \\
& * M\left(g\left(x_{n}^{(2)}\right), g\left(x_{n+1}^{(2)}\right), t\right) \\
& * \cdots * M\left(g\left(x_{n}^{(n)}\right), g\left(x_{n+1}^{(n)}\right), t\right) \\
\geq & \delta_{n}(t) \geq \delta_{n-1}(t-k t) .
\end{aligned}
$$

Next, we claim that

$$
\begin{aligned}
M( & \left.g\left(x_{n}^{(1)}\right), g\left(x_{n+p}^{(1)}\right), t\right) \\
& * M\left(g\left(x_{n}^{(2)}\right), g\left(x_{n+p}^{(2)}\right), t\right) \\
& * \cdots * M\left(g\left(x_{n}^{(n)}\right), g\left(x_{n+p}^{(n)}\right), t\right) \\
\geq & *^{p} \delta_{n-1}(t-k t)
\end{aligned}
$$


for all $t>0$ and $n, p \geq 1$. We prove it by using mathematical induction on $p \geq 1$. If $p=1$, (24) is true for all $n \geq 1$ and all $t>0$ by (23). Suppose that (24) is true for all $n \geq 1$ and all $t>0$ for some $p$, and we are going to prove it is also true for $p+1$. Applying (6), the induction hypothesis, and that $* \geq \cdot$,

$$
\begin{aligned}
M & \left(g\left(x_{n+1}^{(1)}\right), g\left(x_{n+p+1}^{(1)}\right), k t\right) \\
= & M\left(F\left(x_{n}^{(1)}, x_{n}^{(2)}, \ldots, x_{n}^{(n)},\right) F\left(x_{n+p}^{(1)}, x_{n+p}^{(2)}, \ldots, x_{n+p}^{(n)}\right), k t\right) \\
\geq & {\left[M\left(g x_{n}^{(1)}, g x_{n+p}^{(1)}, t\right)\right]^{a_{1}} } \\
& *\left[M\left(g x_{n}^{(2)}, g x_{n+p}^{(2)}, t\right)\right]^{a_{2}} \\
& * \cdots *\left[M\left(g x_{n}^{(n)}, g x_{n+p}^{(n)}, t\right)\right]^{a_{n}} \\
\geq & \left(*^{p} \delta_{n-1}(t-k t)\right)^{a_{1}} \\
& *\left(*^{p} \delta_{n-1}(t-k t)\right)^{a_{2}} \\
& * \cdots *\left(*^{p} \delta_{n-1}(t-k t)\right)^{a_{n}} \\
\geq & \left(*^{p} \delta_{n-1}(t-k t)\right)^{a_{1}} \\
& \cdot\left(*^{p} \delta_{n-1}(t-k t)\right)^{a_{2}} \\
& \cdot \ldots \cdot\left(*^{p} \delta_{n-1}(t-k t)\right)^{a_{n}} \\
\geq & \left(*^{p} \delta_{n-1}(t-k t)\right)^{a_{1}+a_{2}+\cdots+a_{n}} \\
\geq & *^{p} \delta_{n-1}(t-k t) .
\end{aligned}
$$

Arguing in the same way, we arrive at the following:

$$
\begin{aligned}
M( & \left.g\left(x_{n+1}^{(2)}\right), g\left(x_{n+p+1}^{(2)}\right), k t\right), \\
& M\left(g\left(x_{n+1}^{(3)}\right), g\left(x_{n+p+1}^{(3)}\right), k t\right), \ldots, \\
& M\left(g\left(x_{n+1}^{(n)}\right), g\left(x_{n+p+1}^{(n)}\right), k t\right) \\
\geq & *^{p} \delta_{n-1}(t-k t) .
\end{aligned}
$$

Applying the axiom $\left(\mathrm{FM}_{5}\right)$ of a FMS, (18), and the induction hypothesis,

$$
\begin{aligned}
M( & \left.g\left(x_{n}^{(1)}\right), g\left(x_{n+p+1}^{(1)}\right), t\right) \\
= & M\left(g\left(x_{n}^{(1)}\right), g\left(x_{n+p+1}^{(1)}\right),(t+k t-k t)\right) \\
\geq & M\left(g\left(x_{n}^{(1)}\right), g\left(x_{n+1}^{(1)}\right),(t-k t)\right) \\
& * M\left(g\left(x_{n+1}^{(1)}\right), g\left(x_{n+p+1}^{(1)}\right), k t\right) \\
\geq & \delta_{n-1}(t-k t) *\left(*^{p} \delta_{n-1}(t-k t)\right) \\
= & *^{p+1} \delta_{n-1}(t-k t) .
\end{aligned}
$$

Arguing in the same manner, we have

$$
\begin{aligned}
& M\left(g\left(x_{n}^{(2)}\right), g\left(x_{n+p+1}^{(2)}\right), t\right), \\
& M\left(g\left(x_{n}^{(3)}\right), g\left(x_{n+p+1}^{(3)}\right), t\right), \ldots, \\
& M\left(g\left(x_{n}^{(n)}\right), g\left(x_{n+p+1}^{(n)}\right), t\right) .
\end{aligned}
$$

Therefore, (24) is true.
This permits us to show that $\left\{g x_{n}^{(1)}\right\}$ is Cauchy. Suppose that $t>0$ for a given $\epsilon \in(0,1)$.

By the hypothesis, as $*$ is a $t$-norm of $H$-type, there exists $0<\eta<1$ such that $*^{p} a>1-\epsilon$ for all $a \in(1-\eta, 1]$ and for all $p \geq 1$.

By (22), $\lim _{n \rightarrow \infty} \delta_{n}(t)=1$, so there exists $n_{0} \in N$ such that $\delta_{n}(t-k t)>1-\eta$ for all $n \geq n_{0}$.

Hence (24), we get

$$
\begin{aligned}
& M\left(g\left(x_{n}^{(1)}\right), g\left(x_{n+p+1}^{(1)}\right), t\right), \\
& \quad M\left(g\left(x_{n}^{(2)}\right), g\left(x_{n+p+1}^{(2)}\right), t\right), \ldots, \\
& \quad M\left(g\left(x_{n}^{(n)}\right), g\left(x_{n+p+1}^{(n)}\right), t\right)>1-\epsilon
\end{aligned}
$$

for all $n \geq n_{0}$ and $p \geq 1$.

Therefore, $\left\{g x_{n}^{(1)}\right\}$ is a Cauchy sequence. Similarly, we can show that $\left\{g x_{n}^{(2)}\right\},\left\{g x_{n}^{(3)}\right\}, \ldots,\left\{g x_{n}^{(n)}\right\}$ are also Cauchy sequences.

Step 3. Now, we claim that $g$ and $F$ have a tripled coincidence point. Since $X$ is complete, there exists $x^{(1)}, x^{(2)}, \ldots, x^{(n)} \in X$ such that

$$
\begin{gathered}
\lim _{n \rightarrow \infty} g x_{n}^{(1)}=x^{(1)}, \lim _{n \rightarrow \infty} g x_{n}^{(2)}=x^{(2)}, \ldots, \\
\lim _{n \rightarrow \infty} g x_{n}^{(n)}=x^{(n)} .
\end{gathered}
$$

As $g$ is continuous, we have that

$$
\begin{aligned}
& \lim _{n \rightarrow \infty} g g x_{n}^{(1)}=g x^{(1)}, \lim _{n \rightarrow \infty} g g x_{n}^{(2)}=g x^{(2)}, \ldots, \\
& \lim _{n \rightarrow \infty} g g x_{n}^{(n)}=g x^{(n)} .
\end{aligned}
$$

The commutativity of $F$ with $g$ implies that

$$
\begin{aligned}
g g x_{n+1}^{(1)} & =g F\left(x_{n}^{(1)}, x_{n}^{(2)}, \ldots, x_{n}^{(n)}\right) \\
& =F\left(g x_{n}^{(1)}, g x_{n}^{(2)}, \ldots, g x_{n}^{(n)}\right) .
\end{aligned}
$$

By (6) and Remark 8, we get

$$
\begin{gathered}
M\left(g g x_{n+1}^{(1)}, F\left(x^{(1)}, x^{(2)}, \ldots, x^{(n)}\right), k t\right) \\
=M\left(F\left(g x_{n}^{(1)}, g x_{n}^{(2)}, \ldots, g x_{n}^{(n)}\right),\right. \\
\left.F\left(x^{(1)}, x^{(2)}, \ldots, x^{(n)}\right), k t\right) \\
\geq\left[M\left(g g x_{n}^{(1)}, g x^{(1)}, t\right)\right]^{a_{1}}
\end{gathered}
$$




$$
\begin{aligned}
& *\left[M\left(g g x_{n}^{(2)}, g x^{(2)}, t\right)\right]^{a_{2}} \\
& * \cdots *\left[M\left(g g x_{n}^{(n)}, g x^{(n)}, t\right)\right]^{a_{n}} \\
\geq & M\left(g g x_{n}^{(1)}, g x^{(1)}, t\right) * M\left(g g x_{n}^{(2)}, g x^{(2)}, t\right) \\
& * \cdots * M\left(g g x_{n}^{(n)}, g x^{(n)}, t\right) .
\end{aligned}
$$

Letting $n \rightarrow \infty$, we deduce that

$$
\lim _{n \rightarrow \infty} g g x_{n}^{(1)}=F\left(x^{(1)}, x^{(2)}, \ldots, x^{(n)}\right) .
$$

Hence, $F\left(x^{(1)}, x^{(2)}, \ldots, x^{(n)}\right)=g\left(x^{(1)}\right)$.

Similarly, we can show that

$$
\begin{gathered}
F\left(x^{(2)}, x^{(3)}, \ldots, x^{(1)}\right)=g\left(x^{(2)}\right), \\
F\left(x^{(3)}, x^{(4)}, \ldots x^{(2)}\right)=g\left(x^{(3)}\right), \\
\vdots \\
F\left(x^{(n)}, x^{(1)}, \ldots, x^{(n-1)}\right)=g\left(x^{(n)}\right) .
\end{gathered}
$$

So $\left(x^{(1)}, x^{(2)}, \ldots, x^{(n)}\right)$ is $n$-tupled coincidence point of the mappings $F$ and $g$ :

$$
\begin{gathered}
F\left(x^{(1)}, x^{(2)}, \ldots, x^{(n)}\right)=g\left(x^{(1)}\right) \\
F\left(x^{(2)}, x^{(3)}, \ldots, x^{(1)}\right)=g\left(x^{(2)}\right) \\
\vdots \\
F\left(x^{(n)}, x^{(1)}, \ldots, x^{(n-1)}\right)=g\left(x^{(n)}\right) .
\end{gathered}
$$

Step 4. Now, we claim that

$$
\begin{gathered}
F\left(x^{(1)}, x^{(2)}, \ldots, x^{(n)}\right)=x^{(2)} \\
F\left(x^{(2)}, x^{(3)}, \ldots, x^{(1)}\right)=x^{(3)} \\
\vdots \\
F\left(x^{(n)}, x^{(1)}, \ldots, x^{(n-1)}\right)=x^{(1)} .
\end{gathered}
$$

We note that, by condition (6),

$$
\begin{aligned}
& M\left(g x^{(1)}, g x_{n}^{(2)}, k t\right) \\
& =M\left(F\left(x^{(1)}, x^{(2)}, \ldots, x^{(n)}\right),\right. \\
& \left.\quad F\left(x_{n-1}^{(2)}, x_{n-1}^{(3)}, \ldots, x_{n-1}^{(1)}\right), k t\right)
\end{aligned}
$$

$$
\begin{aligned}
\geq & {\left[M\left(g x^{(1)}, g x_{n-1}^{(2)}, t\right)\right]^{a_{1}} } \\
* & {\left[M\left(g x^{(2)}, g x_{n-1}^{(3)}, t\right)\right]^{a_{2}} * \ldots } \\
* & {\left[M\left(g x^{(n)}, g x_{n-1}^{(1)}, t\right)\right]^{a_{n}} } \\
M\left(g x^{(2)}, g x_{n}^{(3)}, k t\right) & \\
=M & \left(F\left(x^{(2)}, x^{(3)}, \ldots, x^{(1)}\right),\right. \\
& \left.F\left(x_{n-1}^{(3)}, x_{n-1}^{(4)}, \ldots, x_{n-1}^{(2)}\right), k t\right) \\
\geq & {\left[M\left(g x^{(2)}, g x_{n-1}^{(3)}, t\right)\right]^{a_{1}} } \\
* & {\left[M\left(g x^{(3)}, g x_{n-1}^{(4)}, t\right)\right]^{a_{2}} * \ldots } \\
* & {\left[M\left(g x^{(1)}, g x_{n-1}^{(2)}, t\right)\right]^{a_{n}} }
\end{aligned}
$$

$$
\begin{gathered}
M\left(g x^{(n)}, g x_{n}^{(1)}, k t\right) \\
=M\left(F\left(x^{(n)}, x^{(1)}, \ldots, x^{(n-1)}\right),\right. \\
\left.F\left(x_{n-1}^{(1)}, x_{n-1}^{(2)}, \ldots, x_{n-1}^{(n)}\right), k t\right) \\
\geq\left[M\left(g x^{(n)}, g x_{n-1}^{(1)}, t\right)\right]^{a_{1}} \\
*\left[M\left(g x^{(1)}, g x_{n-1}^{(2)}, t\right)\right]^{a_{2}} * \cdots \\
*\left[M\left(g x^{(n-1)}, g x_{n-1}^{(n)}, t\right)\right]^{a_{n}} .
\end{gathered}
$$

Let $\beta_{n}(t)=M\left(g x^{(1)}, g x_{n}^{(2)}, t\right) * M\left(g x^{(2)}, g x_{n}^{(3)}, t\right) * \cdots *$ $M\left(g x^{(n)}, g x_{n}^{(1)}, t\right)$ for all $t>0$ and $n \geq 0$.

By (38),

$$
\begin{aligned}
\beta_{n+1}(k t)=M\left(g x^{(1)}, g x_{n+1}^{(2)}, k t\right) \\
* M\left(g x^{(2)}, g x_{n+1}^{(3)}, k t\right) \\
* \cdots * M\left(g x^{(n)}, g x_{n+1}^{(1)}, k t\right) \\
\geq\left(\left[M\left(g x^{(1)}, g x_{n}^{(2)}, t\right)\right]^{a_{1}}\right. \\
*\left[M\left(g x^{(2)}, g x_{n}^{(3)}, t\right)\right]^{a_{2}} \\
\left.* \cdots *\left[M\left(g x^{(n)}, g x_{n}^{(1)}, t\right)\right]^{a_{n}}\right) \\
*\left(\left[M\left(g x^{(2)}, g x_{n}^{(3)}, t\right)\right]^{a_{1}}\right. \\
*\left[M\left(g x^{(3)}, g x_{n}^{(4)}, t\right)\right]^{a_{2}} \\
\left.* \cdots *\left[M\left(g x^{(1)}, g x_{n}^{(2)}, t\right)\right]^{a_{n}}\right) \\
*\left(\left[M\left(g x^{(n)}, g x_{n}^{(1)}, t\right)\right]^{a_{1}}\right. \\
*\left[M\left(g x^{(1)}, g x_{n}^{(2)}, t\right)\right]^{a_{2}} \\
\left.* \cdots *\left[M\left(g x^{(n-1)}, g x_{n}^{(n)}, t\right)\right]^{a_{n}}\right) \\
* \cdots *
\end{aligned}
$$




$$
\begin{aligned}
& =\left(\left[M\left(g x^{(1)}, g x_{n}^{(2)}, t\right)\right]^{a_{1}}\right. \\
& \text { * }\left[M\left(g x^{(1)}, g x_{n}^{(2)}, t\right)\right]^{a_{2}}
\end{aligned}
$$

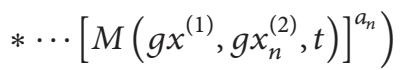

$$
\begin{aligned}
& \text { * }\left(\left[M\left(g x^{(2)}, g x_{n}^{(3)}, t\right)\right]^{a_{1}}\right. \\
& \text { * }\left[M\left(g x^{(2)}, g x_{n}^{(3)}, t\right)\right]^{a_{2}}
\end{aligned}
$$

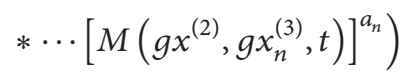

$$
\begin{aligned}
& \text { * }\left(\left[M\left(g x^{(n)}, g x_{n}^{(1)}, t\right)\right]^{a_{1}}\right. \\
& \text { * }\left[M\left(g x^{(n)}, g x_{n}^{(1)}, t\right)\right]^{a_{2}}
\end{aligned}
$$

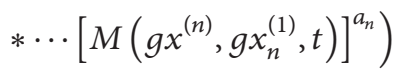

$$
\begin{aligned}
& \geq\left(\left[M\left(g x^{(1)}, g x_{n}^{(2)}, t\right)\right]^{a_{1}}\right. \\
& \cdot\left[M\left(g x^{(1)}, g x_{n}^{(2)}, t\right)\right]^{a_{2}} \\
& \left.\cdot \ldots\left[M\left(g x^{(1)}, g x_{n}^{(2)}, t\right)\right]^{a_{n}}\right) \\
& \text { * }\left(\left[M\left(g x^{(2)}, g x_{n}^{(3)}, t\right)\right]^{a_{1}}\right. \\
& \cdot\left[M\left(g x^{(2)}, g x_{n}^{(3)}, t\right)\right]^{a_{2}} \\
& \left.\cdot \ldots\left[M\left(g x^{(2)}, g x_{n}^{(3)}, t\right)\right]^{a_{n}}\right) \\
& \text { * }\left(\left[M\left(g x^{(n)}, g x_{n}^{(1)}, t\right)\right]^{a_{1}}\right. \\
& \cdot\left[M\left(g x^{(n)}, g x_{n}^{(1)}, t\right)\right]^{a_{2}} \\
& \left.\cdot \ldots\left[M\left(g x^{(n)}, g x_{n}^{(1)}, t\right)\right]^{a_{n}}\right) \\
& =\left[M\left(g x^{(1)}, g x_{n}^{(2)}, t\right)\right]^{a_{1}+a_{2}+\cdots+a_{n}} \\
& \text { * }\left[M\left(g x^{(1)}, g x_{n}^{(2)}, t\right)\right]^{a_{1}+a_{2}+\cdots+a_{n}} \\
& * \cdots *\left[M\left(g x^{(1)}, g x_{n}^{(2)}, t\right)\right]^{a_{1}+a_{2}+\cdots+a_{n}} \\
& \geq M\left(g x^{(1)}, g x_{n}^{(2)}, t\right) * M\left(g x^{(2)}, g x_{n}^{(3)}, t\right) \\
& * M\left(g x^{(n)}, g x_{n}^{(1)}, t\right) \\
& =\beta_{n}(t) \text {. }
\end{aligned}
$$

This proves that $\beta_{n+1}(k t) \geq \beta_{n}(t)$ for all $t>0$ and $n \geq 0$. Repeating this process,

$$
\beta_{n}(t) \geq \beta_{n-1}\left(\frac{t}{k}\right) \geq \beta_{n-2}\left(\frac{t}{k^{2}}\right) \geq \cdots \geq \beta_{0}\left(\frac{t}{k^{n}}\right)
$$

Now, by (40) and (38),

$$
\begin{aligned}
M\left(g x^{(1)}, g x_{n+1}^{(2)}, k t\right) \geq & {\left[M\left(g x^{(1)}, g x_{n}^{(2)}, t\right)\right]^{a_{1}} } \\
& *\left[M\left(g x^{(2)}, g x_{n}^{(3)}, t\right)\right]^{a_{2}} \\
& * \cdots *\left[M\left(g x^{(n)}, g x_{n}^{(1)}, t\right)\right]^{a_{n}} \\
\geq & \beta_{n}(t) \geq \beta_{0}\left(\frac{t}{k^{n}}\right) \\
M\left(g x^{(2)}, g x_{n+1}^{(3)}, k t\right) \geq & {\left[M\left(g x^{(2)}, g x_{n}^{(3)}, t\right)\right]^{a_{1}} } \\
& *\left[M\left(g x^{(3)}, g x_{n}^{(4)}, t\right)\right]^{a_{2}} \\
& * \cdots *\left[M\left(g x^{(1)}, g x_{n}^{(2)}, t\right)\right]^{a_{n}} \\
\geq & \beta_{n}(t) \geq \beta_{0}\left(\frac{t}{k^{n}}\right) \\
& \vdots \\
M\left(g x^{(n)}, g x_{n+1}^{(1)}, k t\right) \geq & {\left[M\left(g x^{(n)}, g x_{n}^{(1)}, t\right)\right]^{a_{1}} } \\
& *\left[M\left(g x^{(1)}, g x_{n}^{(2)}, t\right)\right]^{a_{2}} \\
& * \cdots *\left[M\left(g x^{(n-1)}, g x_{n}^{(n)}, t\right)\right]^{a_{n}} \\
\geq & \beta_{n}(t) \geq \beta_{0}\left(\frac{t}{k^{n}}\right) .
\end{aligned}
$$

Therefore,

$$
\begin{gathered}
M\left(g x^{(1)}, g x_{n+1}^{(2)}, k t\right), M\left(g x^{(2)}, g x_{n+1}^{(3)}, k t\right), \ldots, \\
M\left(g x^{(n)}, g x_{n+1}^{(1)}, k t\right) \geq \beta_{0}\left(\frac{t}{k^{n}}\right)
\end{gathered}
$$

for all $t>0$ and $n \geq 1$.

Since $\lim _{n \rightarrow \infty} \beta_{0}\left(t / k^{n}\right)=1$ for all $t>0$, we have, taking limit in (41), that

$$
\begin{gathered}
\lim _{n \rightarrow \infty} g x_{n+1}^{(2)}=g x^{(1)}, \lim _{n \rightarrow \infty} g x_{n+1}^{(3)}=g x^{(2)}, \ldots, \\
\lim _{n \rightarrow \infty} g x_{n+1}^{(1)}=g x^{(n)} .
\end{gathered}
$$

This shows, using (36), that

$$
\begin{gathered}
F\left(x^{(1)}, x^{(2)}, \ldots, x^{(n)}\right)=g\left(x^{(1)}\right)=\lim _{n \rightarrow \infty} g x_{n}^{(2)}=x^{(2)}, \\
F\left(x^{(2)}, x^{(3)}, \ldots, x^{(1)}\right)=g\left(x^{(2)}\right)=\lim _{n \rightarrow \infty} g x_{n}^{(3)}=x^{(3)}, \\
\vdots \\
F\left(x^{(n-1)}, x^{(n)}, \ldots, x^{(n-2)}\right)=g\left(x^{(n-1)}\right) \\
=\lim _{n \rightarrow \infty} g x_{n}^{(n)}=x^{(n)} .
\end{gathered}
$$

Step 5. Now, we will prove that $x^{(1)}=x^{(2)}=\cdots=x^{(n)}$. Let

$$
\begin{aligned}
\theta(t)= & M\left(x^{(1)}, x^{(2)}, t\right) * M\left(x^{(2)}, x^{(3)}, t\right) \\
& * \cdots * M\left(x^{(n)}, x^{(1)}, t\right)
\end{aligned}
$$

for all $t>0$. 
Using condition (6), we have

$$
\begin{aligned}
& M\left(x^{(1)}, x^{(2)}, k t\right) \\
& =M\left(F\left(x^{(n)}, x^{(1)}, \ldots, x^{(n-1)}\right),\right. \\
& \left.F\left(x^{(1)}, x^{(2)}, \ldots, x^{(n)}\right), k t\right) \\
& \geq\left[M\left(g x^{(n)}, g x^{(1)}, t\right)\right]^{a_{1}} \\
& \text { * }\left[M\left(g x^{(1)}, g x^{(2)}, t\right)\right]^{a_{2}} \\
& * \cdots *\left[M\left(g x^{(n-1)}, g x^{(n)}, t\right)\right]^{a_{n}} \\
& =\left[M\left(x^{(1)}, x^{(2)}, t\right)\right]^{a_{1}} \\
& \text { * }\left[M\left(x^{(2)}, x^{(3)}, t\right)\right]^{a_{2}} \\
& * \cdots *\left[M\left(x^{(n)}, x^{(1)}, t\right)\right]^{a_{n}} \\
& M\left(x^{(2)}, x^{(3)}, k t\right) \\
& =M\left(F\left(x^{(1)}, x^{(2)}, \ldots, x^{(n)}\right),\right. \\
& \left.F\left(x^{(2)}, x^{(3)}, \ldots, x^{(1)}\right), k t\right) \\
& \geq\left[M\left(g x^{(1)}, g x^{(2)}, t\right)\right]^{a_{1}} *\left[M\left(g x^{(2)}, g x^{(3)}, t\right)\right]^{a_{2}} \\
& * \cdots *\left[M\left(g x^{(n)}, g x^{(1)}, t\right)\right]^{a_{n}} \\
& =\left[M\left(x^{(2)}, x^{(3)}, t\right)\right]^{a_{1}} *\left[M\left(x^{(3)}, x^{(4)}, t\right)\right]^{a_{2}} \\
& * \cdots *\left[M\left(x^{(1)}, x^{(2)}, t\right)\right]^{a_{n}} \\
& M\left(x^{(n)}, x^{(1)}, k t\right) \\
& =M\left(F\left(x^{(n-1)}, x^{(n)}, \ldots, x^{(n-2)}\right),\right. \\
& \left.F\left(x^{(n)}, x^{(1)}, \ldots, x^{(n-1)}\right), k t\right) \\
& \geq\left[M\left(g x^{(n-1)}, g x^{(n)}, t\right)\right]^{a_{1}} *\left[M\left(g x^{(n)}, g x^{(1)}, t\right)\right]^{a_{2}} \\
& * \cdots *\left[M\left(g x^{(n-2)}, g x^{(n-1)}, t\right)\right]^{a_{n}} \\
& =\left[M\left(x^{(n)}, x^{(1)}, t\right)\right]^{a_{1}} *\left[M\left(x^{(1)}, x^{(2)}, t\right)\right]^{a_{2}} \\
& * \cdots *\left[M\left(x^{(n-1)}, x^{(n)}, t\right)\right]^{a_{n}} \text {. }
\end{aligned}
$$

Combining all the inequalities, we will have the following:

$\theta(k t)$

$$
\begin{aligned}
= & M\left(x^{(1)}, x^{(2)}, k t\right) * M\left(x^{(2)}, x^{(3)}, k t\right) \\
& * \cdots * M\left(x^{(n)}, x^{(1)}, k t\right) \\
\geq & \left(\left[M\left(x^{(1)}, x^{(2)}, t\right)\right]^{a_{1}} *\left[M\left(x^{(2)}, x^{(3)}, t\right)\right]^{a_{2}}\right. \\
& \left.* \cdots *\left[M\left(x^{(n)}, x^{(1)}, t\right)\right]^{a_{n}}\right) \\
& *\left(\left[M\left(x^{(2)}, x^{(3)}, t\right)\right]^{a_{1}} *\left[M\left(x^{(3)}, x^{(4)}, t\right)\right]^{a_{2}}\right.
\end{aligned}
$$

$$
\left.* \cdots *\left[M\left(x^{(1)}, x^{(2)}, t\right)\right]^{a_{n}}\right)
$$

$* \cdots$

$$
\begin{aligned}
& *\left(\left[M\left(x^{(n)}, x^{(1)}, t\right)\right]^{a_{1}} *\left[M\left(x^{(1)}, x^{(2)}, t\right)\right]^{a_{2}}\right. \\
& \left.* \cdots *\left[M\left(x^{(n-1)}, x^{(n)}, t\right)\right]^{a_{n}}\right) \\
& =\left(\left[M\left(x^{(1)}, x^{(2)}, t\right)\right]^{a_{1}} *\left[M\left(x^{(1)}, x^{(2)}, t\right)\right]^{a_{2}}\right. \\
& \left.* \cdots *\left[M\left(x^{(1)}, x^{(2)}, t\right)\right]^{a_{n}}\right) \\
& *\left(\left[M\left(x^{(2)}, x^{(3)}, t\right)\right]^{a_{1}} *\left[M\left(x^{(2)}, x^{(3)}, t\right)\right]^{a_{2}}\right. \\
& \left.* \cdots *\left[M\left(x^{(2)}, x^{(3)}, t\right)\right]^{a_{n}}\right) \\
& \text { * } \cdots \\
& *\left(\left[M\left(x^{(n)}, x^{(1)}, t\right)\right]^{a_{1}} *\left[M\left(x^{(n)}, x^{(1)}, t\right)\right]^{a_{2}}\right. \\
& \left.* \cdots *\left[M\left(x^{(n)}, x^{(1)}, t\right)\right]^{a_{n}}\right) \\
& \geq\left(\left[M\left(x^{(1)}, x^{(2)}, t\right)\right]^{a_{1}} \cdot\left[M\left(x^{(1)}, x^{(2)}, t\right)\right]^{a_{2}}\right. \\
& \left.\cdot \ldots \cdot\left[M\left(x^{(1)}, x^{(2)}, t\right)\right]^{a_{n}}\right) \\
& *\left(\left[M\left(x^{(2)}, x^{(3)}, t\right)\right]^{a_{1}} \cdot\left[M\left(x^{(2)}, x^{(3)}, t\right)\right]^{a_{2}}\right. \\
& \left.\cdot \ldots \cdot\left[M\left(x^{(2)}, x^{(3)}, t\right)\right]^{a_{n}}\right) \\
& * \\
& \text { * }\left(\left[M\left(x^{(n)}, x^{(1)}, t\right)\right]^{a_{1}} \cdot\left[M\left(x^{(n)}, x^{(1)}, t\right)\right]^{a_{2}}\right. \\
& \left.\cdot \ldots \cdot\left[M\left(x^{(n)}, x^{(1)}, t\right)\right]^{a_{n}}\right) \\
& =\left(\left[M\left(x^{(1)}, x^{(2)}, t\right)\right]^{a_{1}+a_{2}+\cdots+a_{n}}\right) \\
& \text { * }\left(\left[M\left(x^{(2)}, x^{(3)}, t\right)\right]^{a_{1}+a_{2}+\cdots+a_{n}}\right) \\
& * \cdots *\left(\left[M\left(x^{(n)}, x^{(1)}, t\right)\right]^{a_{1}+a_{2}+\cdots+a_{n}}\right) \\
& \geq M\left(x^{(1)}, x^{(2)}, t\right) * M\left(x^{(2)}, x^{(3)}, t\right) \\
& * \cdots * M\left(x^{(n)}, x^{(1)}, t\right)=\theta(t) .
\end{aligned}
$$

We conclude that $\theta(k t) \geq \theta(t)$ implies that $\theta(t) \geq \theta(t / k) \geq$ $\theta\left(t / k^{2}\right) \geq \cdots \geq \theta\left(t / k^{n}\right)$ for all $t>0$ and $n \geq 1$. From (46),

$$
\begin{aligned}
M\left(x^{(1)}, x^{(2)}, k t\right) \geq & {\left[M\left(x^{(1)}, x^{(2)}, t\right)\right]^{a_{1}} } \\
& *\left[M\left(x^{(2)}, x^{(3)}, t\right)\right]^{a_{2}} \\
& * \cdots *\left[M\left(x^{(n)}, x^{(1)}, t\right)\right]^{a_{n}} \\
\geq & M\left(x^{(1)}, x^{(2)}, t\right) * M\left(x^{(2)}, x^{(3)}, t\right)
\end{aligned}
$$




$$
\begin{gathered}
* \cdots * M\left(x^{(n)}, x^{(1)}, t\right) \\
=\theta(t) \geq \theta\left(\frac{t}{k^{n}}\right) \\
M\left(x^{(2)}, x^{(3)}, k t\right) \geq \\
\left.* M\left(x^{(2)}, x^{(3)}, t\right)\right]^{a_{1}} \\
*\left[M\left(x^{(3)}, x^{(4)}, t\right)\right]^{a_{2}} \\
* \cdots *\left[M\left(x^{(1)}, x^{(2)}, t\right)\right]^{a_{n}} \\
\geq M\left(x^{(2)}, x^{(3)}, t\right) * M\left(x^{(3)}, x^{(4)}, t\right) \\
* \cdots * M\left(x^{(1)}, x^{(2)}, t\right) \\
=\theta(t) \geq \theta\left(\frac{t}{k^{n}}\right) \\
\vdots \\
M\left(x^{(n)}, x^{(1)}, k t\right) \geq\left[M\left(x^{(n)}, x^{(1)}, t\right)\right]^{a_{1}} \\
*\left[M\left(x^{(1)}, x^{(2)}, t\right)\right]^{a_{2}} \\
* \cdots *\left[M\left(x^{(n-1)}, x^{(n)}, t\right)\right]^{a_{n}} \\
\geq M\left(x^{(n)}, x^{(1)}, t\right) * M\left(x^{(1)}, x^{(2)}, t\right) \\
* \cdots * M\left(x^{(n-1)}, x^{(n)}, t\right) \\
=\theta(t) \geq \theta\left(\frac{t}{k^{n}}\right) .
\end{gathered}
$$

Letting $n \rightarrow \infty$, we have $\lim _{n \rightarrow \infty} \theta\left(t / k^{n}\right)=1$ for all $t>0$, and it means that $M\left(x^{(1)}, x^{(2)}, k t\right)=M\left(x^{(2)}, x^{(3)}, k t\right)=\cdots=$ $M\left(x^{(n)}, x^{(1)}, k t\right)=1$ for all $t>0$; that is,

$$
x^{(1)}=x^{(2)}=\cdots=x^{(n)} .
$$

The uniqueness of $x^{(1)}$ follows directly from (6).

Remark 12. The uniqueness of the coincidence point of $F$ and $g$ is not always true. For instance, if $F \equiv x_{0}$ is constant and $g \equiv x_{0}$ is also constant, then every $\left(x^{(1)}, x^{(2)}, \ldots, x^{(n)}\right) \in X^{n}$ is a coincidence point of $F$ and $g$.

Consequences. In the proof of the next result, the view of $(X, d)$ as the crisp FMS $\left(X, M_{c}\right.$, min) is used (see Example 6). Here we deduce results for $n$-tupled coincidence point in metric spaces which are generalization of the results of Roldán et al. [11] which are not in a partially ordered set.

Theorem 13. Let $(X, d)$ be a complete metric space and let $F: X^{n} \rightarrow X$ and $g: X \rightarrow X$ be two mappings such that $F\left(X^{n}\right) \subseteq g(X)$ and $g$ is continuous and commuting with $F$. Suppose that $F$ and $g$ satisfy some of the following conditions for all $x^{(1)}, x^{(2)}, \ldots, x^{(n)}, y^{(1)}, y^{(2)}, \ldots, y^{(n)} \in X$;
(1)

$$
\begin{aligned}
d( & \left.F\left(x^{(1)}, x^{(2)}, \ldots, x^{(n)}\right), F\left(y^{(1)}, y^{(2)}, \ldots, y^{(n)}\right)\right) \\
\leq k \max & \left\{d\left(g x^{(1)}, g y^{(1)}\right),\right. \\
& \left.d\left(g x^{(2)}, y^{(2)}\right), \ldots, d\left(g x^{(n)}, g y^{(n)}\right)\right\}
\end{aligned}
$$

for some $k \in(0,1)$,

(2)

$$
\begin{aligned}
& d\left(F\left(x^{(1)}, x^{(2)}, \ldots, x^{(n)}\right), F\left(y^{(1)}, y^{(2)}, \ldots, y^{(n)}\right)\right) \\
& \leq k \max \{ a_{1} d\left(g x^{(1)}, g y^{(1)}\right) \\
&+a_{2} d\left(g x^{(2)}, y^{(2)}\right) \\
&\left.+\cdots+a_{n} d\left(g x^{(n)}, g y^{(n)}\right)\right\}
\end{aligned}
$$

for some $k \in(0,1)$ and some $a_{1}, a_{2}, \ldots, a_{n} \in[0,1 / n]$,

(3)

$$
\begin{aligned}
d( & \left.\left(x^{(1)}, x^{(2)}, \ldots, x^{(n)}\right), F\left(y^{(1)}, y^{(2)}, \ldots, y^{(n)}\right)\right) \\
\leq & a_{1} d\left(g x^{(1)}, g y^{(1)}\right) \\
& +a_{2} d\left(g x^{(2)}, y^{(2)}\right) \\
& +\cdots+a_{n} d\left(g x^{(n)}, g y^{(n)}\right)
\end{aligned}
$$

for some $a_{1}, a_{2}, \ldots, a_{n} \in[0,1]$, such that $\sum_{i=1}^{n} a_{i}<1$.

Then there exists a unique $x \in X$ such that $x=g x=$ $F(x, x, \ldots, x)$.

Proof. (1) Consider $M_{c}$ defined as in Example 6. As $(X, d)$ is complete, then $\left(X, M_{c}, \min \right)$ is a complete FMS. Fix $x^{(1)}, x^{(2)}, \ldots, x^{(n)}, y^{(1)}, y^{(2)}, \ldots, y^{(n)} \in X$ and $t>0$.

Now we are ready to prove (6).

Put $a^{(1)}=a^{(2)}=\cdots=a^{(n)}=1 / n$ and $*=\min$. If $M_{c}\left(g\left(x^{(1)}\right), g y^{(1)}, t\right)=0$ or $M_{c}\left(g\left(x^{(2)}\right), g y^{(2)}, t\right)=0$ or $\cdots$ or $M_{c}\left(g\left(x^{(n)}\right), g y^{(n)}, t\right)=0$, then (6) is obvious.

Suppose that

$$
\begin{gathered}
M_{c}\left(g\left(x^{(1)}\right), g y^{(1)}, t\right)=1, \\
M_{c}\left(g\left(x^{(2)}\right), g y^{(2)}, t\right)=1, \ldots, \\
M_{c}\left(g\left(x^{(n)}\right), g y^{(n)}, t\right)=1 .
\end{gathered}
$$

This means that

$$
\begin{gathered}
d\left(g x^{(1)}, g y^{(1)}\right)<t, d\left(g\left(x^{(2)}\right), g y^{(2)}\right)<t, \ldots, \\
d\left(g\left(x^{(n)}\right), g y^{(n)}\right)<t .
\end{gathered}
$$


Therefore,

$$
\begin{gathered}
t>\max \left\{d\left(g\left(x^{(1)}\right), g y^{(1)}\right), d\left(g\left(x^{(2)}\right), g y^{(2)}\right), \ldots,\right. \\
\left.d\left(g\left(x^{(n)}\right), g y^{(n)}\right)\right\},
\end{gathered}
$$

$k t>k \max \left\{d\left(g x^{(1)}, g y^{(1)}\right), d\left(g x^{(2)}, g y^{(2)}\right), \ldots\right.$,

$$
\begin{gathered}
\left.d\left(g x^{(n)}, g y^{(n)}\right)\right\} \\
\geq d\left(F\left(x^{(1)}, x^{(2)}, \ldots, x^{(n)}\right), F\left(y^{(1)}, y^{(2)}, \ldots, y^{(n)}\right)\right) .
\end{gathered}
$$

Hence, $M_{c}\left(F\left(x^{(1)}, x^{(2)}, \ldots, x^{(n)}\right), F\left(y^{(1)}, y^{(2)}, \ldots, y^{(n)}\right), k t\right)=$ 1 and (6) is also true.

(2) In this case,

$$
\begin{gathered}
d\left(F\left(x^{(1)}, x^{(2)}, \ldots, x^{(n)}\right), F\left(y^{(1)}, y^{(2)}, \ldots, y^{(n)}\right)\right) \\
\leq k\left\{a_{1} d\left(g x^{(1)}, g y^{(1)}\right)+a_{2} d\left(g x^{(2)}, g y^{(2)}\right)\right. \\
\left.+\cdots+a_{n} d\left(g x^{(n)}, g y^{(n)}\right)\right\} \\
\leq k\left\{\frac{1}{n} d\left(g x^{(1)}, g y^{(1)}\right)+\frac{1}{n} d\left(g x^{(2)}, g y^{(2)}\right)\right. \\
\left.+\cdots+\frac{1}{n} d\left(g x^{(n)}, g y^{(n)}\right)\right\} \\
=\frac{k}{n}\left\{d\left(g x^{(1)}, g y^{(1)}\right)+d\left(g x^{(2)}, g y^{(2)}\right)\right. \\
\left.\quad+\cdots+d\left(g x^{(n)}, g y^{(n)}\right)\right\} \\
\leq \frac{k}{n} n \max \left\{d\left(g x^{(1)}, g y^{(1)}\right), d\left(g x^{(2)}, g y^{(2)}\right), \ldots,\right. \\
\left.\quad d\left(g x^{(n)}, g y^{(n)}\right)\right\} \\
=k \max \left\{d\left(g x^{(1)}, g y^{(1)}\right), d\left(g x^{(2)}, g y^{(2)}\right), \ldots,\right. \\
\left.d\left(g x^{(n)}, g y^{(n)}\right)\right\} .
\end{gathered}
$$

(3) If $k=\sum_{i=1}^{n} a_{i}<1$.

$$
\begin{aligned}
d( & \left.F\left(x^{(1)}, x^{(2)}, \ldots, x^{(n)}\right), F\left(y^{(1)}, y^{(2)}, \ldots, y^{(n)}\right)\right) \\
\leq & a_{1} d\left(g x^{(1)}, g y^{(1)}\right)+a_{2} d\left(g x^{(2)}, g y^{(2)}\right) \\
& +\cdots+a_{n} d\left(g x^{(n)}, g y^{(n)}\right) \\
\leq & a_{1} \max \left\{d\left(g x^{(1)}, g y^{(1)}\right), d\left(g x^{(2)}, g y^{(2)}\right), \ldots,\right. \\
& \left.\quad d\left(g x^{(n)}, g y^{(n)}\right)\right\} \\
& +a_{2} \max \left\{d\left(g x^{(1)}, g y^{(1)}\right), d\left(g x^{(2)}, g y^{(2)}\right), \ldots,\right. \\
& \left.\quad d\left(g x^{(n)}, g y^{(n)}\right)\right\} \\
+ & \\
+ & a_{n} \max \left\{d\left(g x^{(1)}, g y^{(1)}\right), d\left(g x^{(2)}, g y^{(2)}\right), \ldots,\right.
\end{aligned}
$$

$$
\begin{gathered}
\left.d\left(g x^{(n)}, g y^{(n)}\right)\right\} \\
=\left(a_{1}+a_{2}+\cdots+a_{n}\right) \\
\cdot \max \left\{d\left(g x^{(1)}, g y^{(1)}\right), d\left(g x^{(2)}, g y^{(2)}\right), \ldots,\right. \\
\left.d\left(g x^{(n)}, g y^{(n)}\right)\right\} \\
=k \max \left\{d\left(g x^{(1)}, g y^{(1)}\right), d\left(g x^{(2)}, g y^{(2)}\right), \ldots,\right. \\
\left.d\left(g x^{(n)}, g y^{(n)}\right)\right\} .
\end{gathered}
$$

Remark 14. We can obtain Theorems 11 and 15 due to Roldán et al. [11] as corollaries, if we establish our main result for the mappings $F: X \times X \times X \rightarrow X$ and $g: X \rightarrow X$.

Applications. Now we are ready to establish application.

Lipschitzian Systems. Let $f_{1}, f_{2}, \ldots, f_{n}: R \rightarrow R$ be Lipschitzian mappings and let $\beta_{1}, \beta_{2}, \ldots, \beta_{n} \in R$ be real numbers. Define $h: R \rightarrow R$ as $h(x)=\beta_{1} f_{1}(x)+\beta_{2} f_{2}(x)+$ $\cdots+\beta_{n} f_{n}(x)$ for all $x \in R$. Then $h$ is another Lipschitzian mapping and $k_{h} \leq\left|\beta_{1}\right| k_{f_{1}}+\left|\beta_{2}\right| k_{f_{2}}+\cdots+\left|\beta_{n}\right| k_{f_{n}}$. Obviously, if $K=\left|\beta_{1}\right| k_{f_{1}}+\left|\beta_{2}\right| k_{f_{2}}+\cdots+\left|\beta_{n}\right| k_{f_{n}}<1$, then $h$ is a contraction, so there exists a unique $x^{(1)} \in R$ such that $h_{x^{(1)}}=x^{(1)}$.

Next, define $F: R^{n} \rightarrow R$ as

$$
\begin{aligned}
F & \left(x^{(1)}, x^{(2)}, \ldots, x^{(n)}\right) \\
& =\beta_{1} f_{1}\left(x^{(1)}\right)+\beta_{2} f_{2}\left(x^{(2)}\right)+\cdots+\beta_{n} f_{n}\left(x^{(n)}\right)
\end{aligned}
$$

for all $x^{(1)}, x^{(2)}, \ldots, x^{(n)} \in R$. It is clear that $F\left(x^{(1)}, x^{(1)}, \ldots, x^{(1)}\right)=h x^{(1)}$ for all $x^{(1)} \in R$. Furthermore,

$$
\begin{aligned}
& d\left(F\left(x^{(1)}, x^{(2)}, \ldots, x^{(n)}\right), F\left(y^{(1)}, y^{(2)}, \ldots, y^{(n)}\right)\right) \\
& \quad \leq \sum_{i=1}^{n}\left|\beta_{i}\right|\left|f_{i}\left(x^{(i)}\right)-f_{i}\left(y^{(i)}\right)\right| \\
& \quad \leq \sum_{i=1}^{n}\left|\beta_{i}\right| k_{f_{i}}\left|x^{(i)}-y^{(i)}\right| \\
& \quad \leq \operatorname{Kaxax}_{1 \leq j \leq n}\left(x^{(j)}, y^{(j)}\right) .
\end{aligned}
$$

If $K<1$, then $F$ verifies (6) with $g\left(x^{(1)}\right)=x^{(1)}$ for all $x^{(1)} \in R$.

Corollary 15. Let $f_{1}, f_{2}, \ldots, f_{n}: R \rightarrow R$ be Lipschitzian mappings on $R$ (provided with the Euclidean metric) and let $\beta_{1}, \beta_{2}, \ldots, \beta_{n} \in R$ such that $\left|\beta_{1}\right| k_{f_{1}}+\left|\beta_{2}\right| k_{f_{2}}+\cdots+\left|\beta_{n}\right| k_{f_{n}}<1$. Then the system

$$
\begin{gathered}
\beta_{1} f_{1}\left(x^{(1)}\right)+\beta_{2} f_{2}\left(x^{(2)}\right)+\cdots+\beta_{n} f_{n}\left(x^{(n)}\right)=\left(x^{(1)}\right), \\
\beta_{1} f_{1}\left(x^{(2)}\right)+\beta_{2} f_{2}\left(x^{(3)}\right)+\cdots+\beta_{n} f_{n}\left(x^{(1)}\right)=\left(x^{(2)}\right) \\
\vdots \\
\beta_{1} f_{1}\left(x^{(n)}\right)+\beta_{2} f_{2}\left(x^{(1)}\right)+\cdots+\beta_{n} f_{n}\left(x^{(n-1)}\right)=\left(x^{(n)}\right)
\end{gathered}
$$


has a unique solution, which is $\left(x^{(1)}, x^{(1)}, \ldots, x^{(1)}\right)$, where $x^{(1)}$ is the only real solution of $\beta_{1} f_{1}\left(x^{(1)}\right)+\beta_{2} f_{2}\left(x^{(2)}\right)+\cdots+$ $\beta_{n} f_{n}\left(x^{(n)}\right)=\left(x^{(1)}\right)$.

An Integral System. Let $a, b \in R$ with $a<b$ and let $I=$ $[a, b]$. Consider $X=\mathfrak{七}^{1}(I)$ with the distance $d_{1} \cdot(f, g)=$ $\int_{I}|f(t)-g(t)| d t$, where $\int$ represents the Lebesgue integral. It is well known that $\left(\mathrm{E}(I), d_{1}\right)$ is a complete metric space. Let $k, \beta_{1}, \beta_{2}, \ldots, \beta_{n} \in R$, be real numbers and let $G: R^{n} \rightarrow R$ be a mapping verifying $G(0,0, \ldots, 0)=0$, and

$$
\left|G_{x^{(1)}, x^{(2)}, \ldots, x^{(n)}}-G_{y^{(1)}, y^{(2)}, \ldots, y^{(n)}}\right| \leq k \sum_{i=1}^{n} \beta_{i}\left|x^{(i)}-y^{(i)}\right|
$$

for all $\left(x^{(1)}, x^{(2)}, \ldots, x^{(n)}\right),\left(y^{(1)}, y^{(2)}, \ldots, y^{(n)}\right) \in R^{n}$

If $A \in R$, we want to find functions $f_{1}, f_{2}, \ldots, f_{n} \in \mathbb{L}^{1}(I)$ such that

$$
f_{i}(x)=A+\int_{[a, x]} G\left(f_{i}(t), f_{i+1}(t), \ldots, f_{i+n-n}(t)\right) d t
$$

holds for all $x \in I, i=1,2, \ldots, n$.

For all $f_{1}, f_{2}, \ldots, f_{n} \in \mathfrak{七}^{1}(I)$ and all $x \in I$, define

$$
\begin{aligned}
& F_{f_{1} f_{2} \cdots f_{n}}(x) \\
& \quad=A+\int_{[a, x]} G\left(f_{1}(t), f_{2}(t) \cdots f_{n}(t)\right) d t .
\end{aligned}
$$

On the one hand, it is not difficult to prove that $F_{f_{1} f_{2} \cdots f_{n}} \in$ $\mathfrak{E}^{1}(I)$; hence $F: \mathbb{L}^{1}(I)^{n} \rightarrow \mathbb{L}^{1}(I)$ is well defined. On the other hand,

$$
\begin{aligned}
& d_{1}\left(F_{f_{1} f_{2} \cdots f_{n}}, F_{g_{1} g_{2} \cdots g_{n}}\right) \\
& \quad=\int_{I}\left|F_{f_{1} f_{2} \cdots f_{n}}(x)-F_{g_{1} g_{2} \cdots g_{n}}(x)\right| d x \\
& \quad \leq \int_{I}\left(\int_{[a, x]} \mid G\left(f_{1}(t), f_{2}(t) \cdots f_{n}(t)\right)\right. \\
& \quad \leq \int_{I}\left(\int_{[a, x]} k \sum_{i=1}^{n} \beta_{i}\left|f_{i}(t)-g_{i}(t)\right| d t\right) d x \\
& \quad \leq k \sum_{i=1}^{n} \beta_{i} \int_{I}\left(\int_{I}\left|f_{i}(t)-g_{i}(t)\right| d t\right) d x \\
& \quad=k \sum_{i=1}^{n} \beta_{i} \int_{I} d_{1}\left(f_{i}, g_{i}\right) d x \\
& \quad=k(b-a) \sum_{i=1}^{n} \beta_{i} d_{1}\left(f_{i}, g_{i}\right) .
\end{aligned}
$$

If we suppose that $K=k(b-a)\left(\beta_{1}, \beta_{2}, \ldots, \beta_{n}\right)<1$, then $F$ verifies (6) with $g(f)=f$ for all $f \in \mathfrak{七}^{1}(I)$. Then system (62) has a unique solution, which is of the form $\left(f_{0}, f_{0}, \ldots, f_{0}\right)$, where $f_{0} \in \mathrm{E}^{1}(I)$ is the only solution of the equation

$$
f_{0}(x)=A+\int_{[a, x]} G\left(f_{0}(t), f_{0}(t), \ldots, f_{0}(t)\right) d t
$$

for all $x \in I$.

\section{Conflict of Interests}

The authors declare that there is no conflict of interests regarding the publication of this paper.

\section{Acknowledgments}

The first author (P. P. Murthy) is thankful to University Grants Commission, New Delhi, India, for financial assistance through Major Research Project File no. 42-32/2013 (SR). Also the authors are grateful to the referees for their valuable comments and suggestions to improve this paper.

\section{References}

[1] T. G. Bhaskar and V. Lakshmikantham, "Fixed point theorems in partially ordered metric spaces and applications," Nonlinear Analysis. Theory, Methods \& Applications, vol. 65, no. 7, pp. 13791393, 2006.

[2] A. Roldán, J. Martínez-Morenoa, C. Roldán, and Y. J. Cho, "Multidimensional coincidence point results for compatible mappings in partially ordered fuzzy metric spaces," Fuzzy Sets and Systems, vol. 251, pp. 71-82, 2014.

[3] A. Roldán, J. Martínez-Moreno, and C. Roldán, "On interrelationships between fuzzy metric structures," Iranian Journal of Fuzzy Systems, vol. 10, pp. 133-150, 2013.

[4] D. J. Guo and V. Lakshmikantham, "Coupled fixed points of nonlinear operators with applications," Nonlinear Analysis. Theory, Methods \& Applications, vol. 11, no. 5, pp. 623-632, 1987.

[5] V. Berinde and M. Borcut, "Tripled fixed point theorems for contractive type mappings in partially ordered metric spaces," Nonlinear Analysis. Theory, Methods \& Applications, vol. 74, no. 15, pp. 4889-4897, 2011.

[6] A. Sharma, "Results on $n$-tupled fixed points in complete asymptotically regular metric spaces," Journal of the Egyptian Mathematical Society, vol. 22, no. 3, pp. 440-445, 2014.

[7] P. Kumam, J. Martínez-Moreno, A.-F. Roldán-López-de-Hierro, and C. Roldán-López-de-Hierro, "Berinde-Borcut tripled fixed point theorem in partially ordered (intuitionistic) fuzzy normed spaces," Journal of Inequalities and Applications, vol. 2014, article no. 47, 2014.

[8] M. Imdad, A. Sharma, and A. Erduran, "Generalized MeirKeeler type $n$-tupled fixed point theorems in ordered partial metric spaces," Fixed Point Theory and Applications, vol. 2014, article 114, 2014.

[9] M. Imdad, A. H. Soliman, B. S. Choudhury, and P. Das, "On $n$-tupled coincidence point results in metric spaces," Journal of Operators, vol. 2013, Article ID 532867, 8 pages, 2013.

[10] A. H. Soliman, M. Imdad, and A. Sharma, "Results on n-tupled coincidence points in generalized complete metric spaces," In Press.

[11] A. Roldán, J. Martínez-Moreno, and C. Roldán, “Tripled fixed point theorem in fuzzy metric spaces and applications," Fixed Point Theory and Applications, vol. 2013, article 29, 2013.

[12] O. Hadic and E. Pap, Fixed Point Theory in Probabilistic Metric Spaces, Kluwer Academic, Dordrecht, The Netherlands, 2001.

[13] I. Kramosil and J. Michálek, "Fuzzy metrics and statistical metric spaces," Kybernetika, vol. 11, no. 5, pp. 336-344, 1975.

[14] A. George and P. Veeramani, "On some results in fuzzy metric spaces," Fuzzy Sets and Systems, vol. 64, no. 3, pp. 395-399, 1994. 

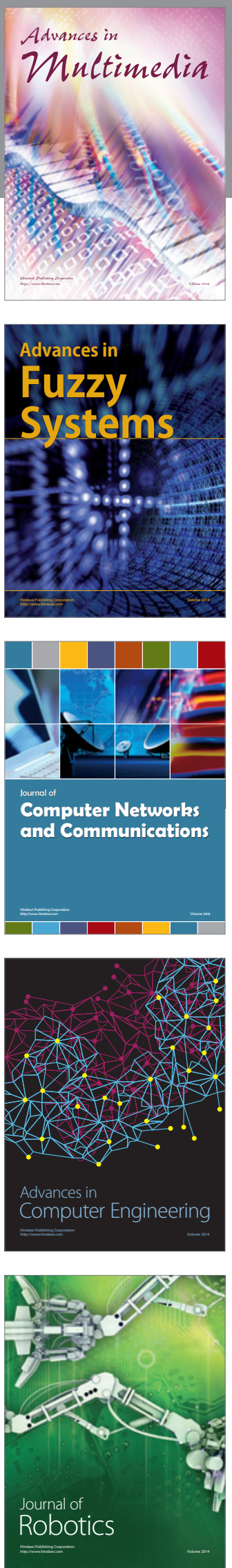

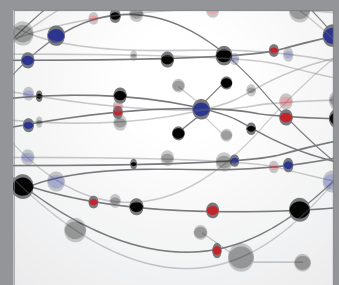

The Scientific World Journal
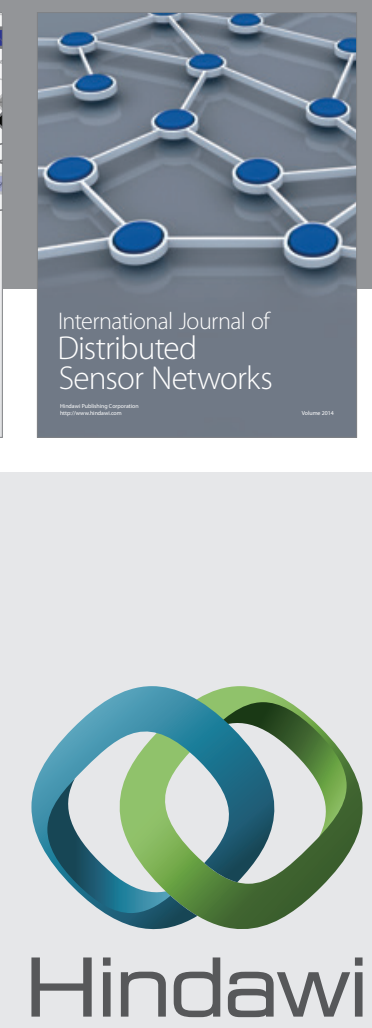

Submit your manuscripts at

http://www.hindawi.com
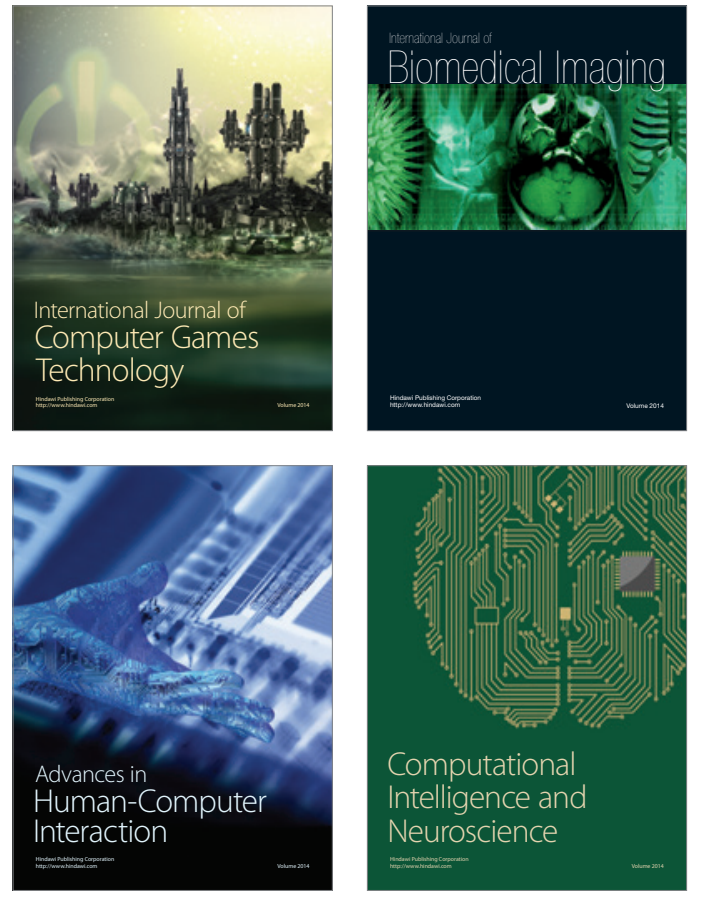
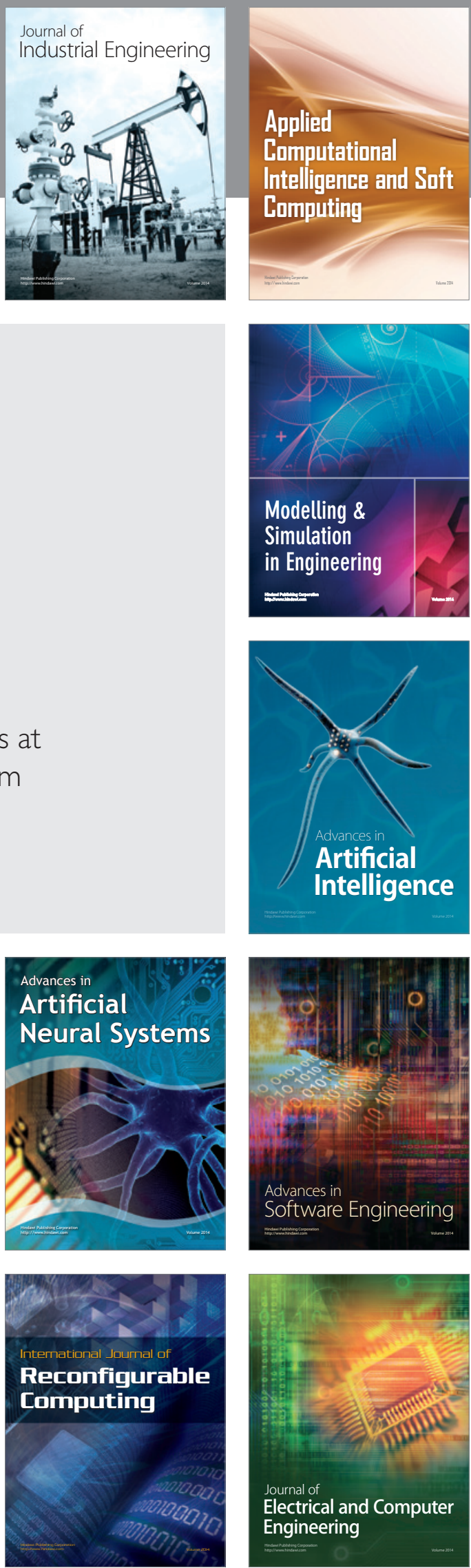